\title{
BREATHING AND BREATHLESSNESS IN CLINIC AND CULTURE: USING CRITICAL MEDICAL HUMANITIES TO BRIDGE AN EPISTEMIC GAP
}

\author{
Jane Macnaughton and Havi Carel
}

\section{Introduction: Critical Medical Humanities and Somatic Illness}

A CENTRAL TENET OF critical medical humanities is the claim that biomedicine does not hold all the keys to understanding the experience of illness, how responses to treatment are mediated, or how outcomes and prognosis are revealed over time. We further suggest that biomedicine cannot wholly explain how illness may be expressed physiologically. So much that influences that expression derives from cultural context, emotional response, and how illness is interpreted and understood that this knowledge cannot be exhausted with the tools of biomedicine.

In as much as it has focused on clinical medicine, medical humanities has tended to concentrate on the manner in which symptoms are presented and discussed by clinician and patient, how potential diagnoses are handled, and how prognoses might be delivered and received. What the field has not interrogated or influenced, until recently, has been bioscientific ways of thinking about how clinical conditions are understood and how research into treatment and management is conceived.

The 'Hearing the Voice' project, ${ }^{1}$ led by one of this volume's editors and involving a number of contributors, is the first project, as far as we are aware, that has attempted specifically to extend the gaze of medical humanities from the clinical interaction to critically examining the evidence base that underlies that interaction. That project's main intersection with clinical medicine is in psychiatry; it studies the phenomenon of auditory verbal hallucination.

This chapter, nested within a section on the body and the senses, emerges from the 'Life of Breath' project, which seeks to explore breathing and breathlessness. These phenomena, we suggest, are similarly complex, poorly understood and often unproductively reduced to their physical components. ${ }^{2}$ It is, to the best of our knowledge, one of the first attempts to apply medical humanities understanding and approaches to the study of 'somatic' phenomena - breathing and breathlessness - with a view to challenging and broadening the evidence base on which breathing symptomatology is addressed clinically. 
In this chapter, we examine breathing and breathlessness as phenomena pregnant with historical, cultural and existential meanings that are often overlooked in the clinical context. We argue that this represents an epistemic gap: an apparently unbridgeable mismatch of understanding not only of knowledge but also of how that knowledge might be obtained, between the clinic and the person who experiences breathlessness. We go on to propose how critical medical humanities may help bridge at least some aspects of this gap by looking at key issues relating to breathlessness that clinicians are grappling with in public health, clinical diagnosis and treatment, and in clinical research. Our focus here is on how a critical medical humanities approach might influence current and future thinking on modelling neurophysiological mechanisms underpinning breathlessness.

In the clinical areas we consider, breathing and breathlessness are comparatively invisible. This is one issue we aim to address in the project. However, given the deep human significance of these phenomena, there is a marked lack of humanities research too. Most of what we find in the humanities literature concerning breathing and breathlessness derives from narrative forms (in Angela Woods's formulation) and are understood or interpreted in relation to larger cultural narratives. ${ }^{3}$ We briefly review some of these, suggesting that there is a need also for phenomenological, non-narrative accounts and practices. We conclude by tentatively exploring what some of these might be in a clinical setting with specific reference to how breath might be understood using novel imaging techniques.

\section{The Multi-dimensionality of Breath}

Breathing is literally at the centre of our bodies; it is essential to life. Much of the time we are unaware of it, in the same way that we cannot feel our heart beating or our stomach digesting food. However, if we call upon the body to do more physical work, breathing becomes laboured, eventually leading to breathlessness; in such situations our breath becomes the focus of attention until it returns to normal.

Breathing is also affected by extremes of emotion: surprise or horror make us gasp, hearty laughter leaves us gasping for breath, crying involves involuntary short, sharp inhalations. What is perhaps less apparent about breath in these everyday experiences is that it is the only bodily site (other than the skin) where interior and exterior spaces are in constant exchange. We breathe in the air and whatever it contains, extract the oxygen we need, and expel carbon dioxide. The air around us, with its pollutants, odours, humidity and heat, becomes internalised briefly, making us beings who are not only in the world, but also of it.

These observations about the everyday experience of breathing point towards the potential for a critical medical humanities approach in this area. The very idea of breath is suffused with metaphor. Breath literally takes place in the chest, the centre of our body; metaphorically it is the core of life; our first and last breaths mark life's beginning and end, and breathing continuously happens throughout life. Breathing is richly modulated by emotional experience, be it pleasurable or painful. 
Our existence, consciously or unconsciously, is influenced by the equilibrium between our bodies and the external world. This is nowhere more significantly experienced than through breath.

However, biomedicine does not acknowledge the ways in which everyday experience and its meanings are implicated in breathing, and thus neglects to incorporate this rich vein into its understanding of breathlessness. Metaphor, emotion and the spiritual and existential dimensions are not part of the language of the clinic, but are a central part of the experience of the patient. As Woods has pointed out, medical humanities has tended to prioritise narrative accounts of patient experience in presenting alternative accounts of illness. ${ }^{4,5}$ Non-narrative aspects of experience are not easy to articulate, but this task is crucial to a comprehensive understanding of breathlessness since it is acknowledged by clinicians and by those who experience breathlessness, that emotion and belief have a profound impact upon the severity of this problem.

Before we set out, we must also ask: what is it that medical humanities wishes to achieve? There is no single answer to this question. Historically, this field was inspired in the US in the 1960s by concerns about healthcare delivery and education. 'Depersonalisation', 'the centrality of molecular biology' and the 'teaching of mechanistic medicine' were the key problems identified. ${ }^{6}$ More recently, engaging more fully with humanities and social science scholarship (at least in the UK) has led the field to focus more upon the generation of new knowledge for its own sake, rather than as a reaction to problems with clinical medicine. It has striven to become academically robust and to go beyond the idea of helping to improve healthcare or to provide an explanatory bridge by which the arts and humanities might be used to convey complex clinical science to patients.

If the field takes an exclusively instrumental approach to serving the ends of clinical research, care and practice, it will be difficult to avoid remaining embedded within a clinical culture that itself remains unexamined. ${ }^{7}$ If clinical culture is viewed from the external vantage point of the humanities, however, entire new vistas may become visible, and thus be opened for critical examination.

The phenomenological concept of habitus is a good illustration of this point. In his analysis of the concept, philosopher Dermot Moran speaks of 'bodily habitus': 'Memories, skills, and practical abilities are literally incorporated in the body, in the way we hold ourselves, move our bodies, walk, sit, eat, look weary, adopt a defeated air, and so on. ${ }^{8}$ The contexts within which we find ourselves, our physical surroundings, and the ways in which the body is acted upon by those surroundings, on this view, actually change the body. The body is not just a neutral object whose objective measures may be taken to be the same, regardless of context.

The idea that a clinical context may itself imbue its subjects with ways of moving, talking and sitting is alien to clinical culture but requires thorough examination and reflection. This insight alone is enough to justify the critical role of medical humanities. But this chapter also offers a critical medical humanities that is interested in helping improve breathless patients' care. The reflective movement we propose to undertake here is from the clinic to critical medical humanities and then back to the clinic, in the 
form of intervention, education, policy change and ultimately improvement of patient care and experience.

This critical arc is one of continuous engagement that does not end after one iteration. Thinking concretely about breathless patients, whilst taking the notion of habitus as a starting point, it seems crucial to explore patients' own memories and skills in order to understand their predicament, but also to recognise that this exploration cannot end by simply noting patients' responses. The aim is to help both patients and those who are trying to develop new approaches to the management of breathlessness. Of course, as we suggest above, there is a danger in committing ourselves as medical humanities scholars to align with the ends of medicine: we may lose our critical framework as we become embroiled in the need to support such work. We believe that this risk is averted by the focus on the iterative process, which does not end in the clinic but also points to new research directions in the humanities and social sciences.

Breathlessness is a condition we all know something about, as we have all experienced breathlessness either as part of normal life or as an abnormal manifestation of one of the common diseases of which breathlessness is a symptom, such as asthma, heart failure, chronic obstructive pulmonary disease (COPD) or lung cancer. We claim that normal breathlessness differs substantially from abnormal breathlessness in its many forms in clinical contexts, where it is regarded as a 'symptom'.

This led us to identify an epistemic gap between clinical knowledge and two other interlinked kinds of knowledge: the broader cultural knowledge within which clinical knowledge is tacitly nested and the idiosyncratic personal experience of breathlessness, also informed by culture and later by encounters in the clinic. The interplay between the three epistemic domains is complex, and made more so by the differences in epistemic authority, credibility and power relations, both within each domain and between the various domains. ${ }^{9}$ This approach not only highlights an issue that was clear to William James: that psychological situatedness is an important determinant of how we might interpret experience. ${ }^{10}$ It also allows us to stress the crucial importance of an interdisciplinary approach to understanding this epistemic gap and how it might be bridged.

We suggest that people who experience breathlessness filter that experience through a rich set of influences that have a long cultural history and determine its seriousness for them. But as they approach clinical services, this lay understanding comes up against bioscience; it is met by a particular series of prescribed questions against which the now-patient must assess their breathlessness and through which their understanding begins to change. That change may be temporary, until they leave the clinic and return to their own environment. But the change may be more deep-seated.

For example, the patient may now adopt a deficit approach to her breathlessness, having been shown through lung function tests (LFTs) what percentage of predicted lung function she has. She might feel that she has failed the test and begin to experience her breathlessness as more anomalous and shameful than before. An interdisciplinary approach is required here because coming to grips with such complex processes requires not only cultural, literary, historical and philosophical examination, but also 
social scientific understanding of how the clinic works as a habitus that may challenge and change lay perceptions. We need this approach in order to appreciate the ways in which the culture of medicine plays a role in creating the objects of its concern.

It is not usual for clinicians to admit humanities or social science evidence into clinical work and policy deliberations. ${ }^{11}$ Hence the first step for us is to identify areas of need that are not answered by a biomedical approach and to examine ways of approaching these needs from a multi-disciplinary and critical medical humanities approach. There are three such areas of need in the domain of breathlessness. They are interlinked, but it will be useful to deal with them separately, as they relate to three key areas of activity: public health, clinical diagnosis and treatment, and clinical research.

\section{Breathlessness and Public Health}

Breathlessness is a key symptom in a number of common and serious diseases, such as heart failure and lung cancer, as well as in COPD, a condition with an increasing global prevalence owing to its association with smoking. Air pollution has been an important cause historically, and women in developing countries often develop COPD as a result of time spent poring over cooking fires. ${ }^{12}$ The World Health Organisation currently ranks COPD as the fourth most common cause of death in developed countries and it is estimated that it will become the third largest global killer by $2020 .^{13}$ COPD affects an estimated 3.7 million people in the UK but crucially only 900,000 of them are aware of having the condition. ${ }^{14}$

Thus breathlessness and COPD are of urgent interest to public health. In particular, the hidden burden of disease - that is, the ways in which it exacts a cost from the person suffering from the disease and from society - is of concern and has been the subject of recent analysis. Gysels and Higginson have described the symptom of breathlessness, and the patients suffering from it, as 'invisible'. This invisibility stems from the fact that breathlessness is a condition that usually has an insidious onset and is often attributed by those who experience it to ageing, lack of exercise, or smoking. ${ }^{15}$ The stigma associated with smoking is also a factor encouraging people to hide their condition or its severity. Clinicians, who are increasingly less likely to visit people in their own homes, are unaware of the complex needs, limitations and adjustments required when living with breathlessness. ${ }^{16}$

The invisibility of breathlessness has a social element but is also political and economic in the clinical context. Smoking is a key aetiological factor in the most common diseases leading to chronic breathlessness, and in developed countries smoking prevalence is now pooled in the lowest socioeconomic groups. Thus those who have power to determine priorities in terms of research spending or treatment do not tend to have day-to-day experience or knowledge of the lives of people with conditions like COPD. Partly in consequence, despite its high prevalence and high levels of mortality, COPD has received little attention from clinical researchers and pharmaceutical companies. ${ }^{17}$ 
The reasons for this neglect are significant for our medical humanities analysis. Breathlessness is something that people expect they will experience more the older they get, or as they gain weight or become less fit. Those who smoke think breathlessness is a natural result of smoking and do not necessarily associate it with the development of a disease. The lack of investment in research is also linked to the idea that breathlessness associated with COPD is a self-inflicted problem, and that it is largely irreversible. Unlike asthma, which has been relatively well researched, COPD cannot be modified by medication, only relieved. Once established, its course is slowly progressive even if the patient stops smoking. ${ }^{18}$ If clinicians and researchers - and indeed the pharmaceutical industry - feel there is likely to be nothing achieved in seeking new therapeutic approaches, research funding tends not to flow in this direction. This neglect of breathlessness has a deeper significance in relation to clinical problems of definition and uncertainty about what is to be treated: the underlying condition or the experienced symptom. We now move on to this issue.

\section{Breathlessness in the Clinic}

Breathlessness is a symptom, not a disease. The traditional clinical approach to dealing with a symptom is to find out what is causing it, treat it and wait for the patient to improve. This rarely happens in chronic breathlessness. As Johnson, Currow and Booth argue, chronic breathlessness frequently results from incurable, often long-term, progressive conditions, and the symptom persists despite treatment of the underlying condition. ${ }^{19}$ They have termed such breathlessness 'refractory' and suggest that the attitude of clinicians and patients towards it is one of 'nothing more can be done,' leading to hopelessness and lack of attention to the symptom from both parties. Patients may no longer report increasing distress to their doctors; clinicians may fail to ask about the problems caused by the breathlessness, as they feel unable to help. The problem of invisibility is compounded by a sense of helplessness. The response to this has been, in part, to look to deeper understanding of the pathophysiological and neurophysiological mechanisms associated with breathlessness in order to seek possible pharmacological or other approaches to alleviating the symptom.

\section{Clinical Research Developments: the Neuroscience of Breathlessness}

This takes us to our final field of clinical interest and development: the clinical science of breathlessness. The American Thoracic Society's 2012 'Update on the Mechanisms, Assessment, and Management of Dyspnea' notes considerable concern about the lack of treatment for dyspnoea (pathological breathlessness) itself. ${ }^{20}$ The statement notes that there are still no drugs for which relief of dyspnoea per se is an approved indication (as opposed to approval for treatments of diseases in which dyspnoea is the prominent symptom). ${ }^{21}$ The main treatment options for breathlessness are opioid medications, which depress breathing, smoking cessation where relevant, and pulmonary rehabilitation. 
Pulmonary rehabilitation aims to teach and encourage regular exercise, which increases patients' fitness and reduces their breathlessness. The programme also includes health education on relevant issues such as panic attacks (common in respiratory conditions), stress management and diet. Pulmonary rehabilitation seems to work for those with pathological breathlessness, as it reduces the feeling of breathlessness, but there is no evidence that it actually improves lung function in important conditions such as COPD (although it does improve fitness). ${ }^{22}$ It is important to note the crucial role of perception in breathlessness. Further confusion for clinicians comes from the fact that patients may experience severe breathlessness with mild disease (as measured by spirometry) and vice versa. ${ }^{23}$ Clinical research has therefore turned its attention from a focus on the body (the lungs and muscles of respiration) to the brain, and has started to investigate the sites of cognitive and affective processes involved in the complex experience of breathlessness.

One of the fascinating aspects of breathing is that it is under both involuntary (autonomic) and voluntary control. It is possible to hold one's breath for a while, even though the autonomic system will eventually override this voluntary action. Therefore there are potentially a number of sites that might be important in the control of breathing, including brain stem and cortical areas. Herigstad and colleagues reviewed a series of papers that revealed at least nine areas involved in the voluntary control of breathing, including cortico-limbic structures that also subserve sensations such as thirst, hunger and pain, and the amygdala (also part of the limbic system) which deals with memory and emotions. ${ }^{24}$

Such neuroimaging studies have the potential to help delineate sensory from affective components of breathlessness and improve understanding of how emotional and cognitive processes affect not only the perception but also the pathophysiology of breathlessness. This means that the search to find new mechanisms by which breathlessness might be relieved through new drugs is on. Claudine Peiffer, for example, is using current neurological understanding to propose that stimulating a sense of relief or pleasantness at the site where the sensation of breathlessness is processed centrally might be a productive direction for this research. ${ }^{25}$

We have explored key clinical concerns in relation to breathlessness for a reason. Our experience in medical humanities suggests it is not easy to engage clinicians who have no particular reason to acknowledge that our field may have anything useful to offer their practice or research. It is, therefore, our responsibility to explain how our work might potentially contribute. The aim is to enter into a dialogue in which the issues we have explored might act as a starting point to stimulate mutual exploration. Critically engaged medical humanities research of the kind we are setting out on in the 'Life of Breath' cannot be carried out without the willing participation of clinicians working alongside humanities and social science researchers, who may have different disciplinary interests but are all committed to answering some common questions. Our discussion above reveals that a crucial field of inquiry in clinical studies of breathlessness is the neuroscientific understanding of breathlessness, and asking how this will support potential work in discovering new methods of treatment. We now turn to some concrete examples of gap-bridging work in the domain of breathlessness. 


\section{Bridging the Epistemic Gap 1: Influencing the Modelling of Breathlessness}

Clinical scientists recognise that there are problems with some of their approaches. They have identified a need for sophisticated work on the phenomenology of breathlessness in order that research on neurophysiological mechanisms and brain mapping can be more accurate and perhaps more successfully lead to potential new treatments. Herigstad notes, 'future research should [. . . employ predictive, and thus testable, models of brain function rather than aimlessly searching for static "blobs" of activation., ${ }^{26}$

In their 2012 review, the American Thoracic Society states that 'more than an an time in the past there is a need for interdisciplinary approaches to research into dyspnea mechanisms and actions that will accelerate translation of research findings into clinical practice. ${ }^{27}$ This call for interdisciplinary work does not explicitly include medical humanities, but there is clearly a need for greater understanding of how the experience of breathlessness is created in the conscious experience of the breathless person.

What a medical humanities approach can contribute here are some pieces of the puzzle, as well as a general framework through which to think through these issues. For example, a phenomenological framework posits the essential indivisibility of experience, which is not acknowledged in the clinical context. Research on mapping the brain areas involved in breathlessness is taking pain studies as its model. ${ }^{28}$ It is recognised by some researchers that such a focus might distract researchers from uncovering the complex sensori-emotional mechanisms that are unique to breathlessness, but nevertheless, the 'pain matrix' has a pervasive hold in this area. ${ }^{29}$ There are two main critiques our approach might make to this, which we can then use to offer constructive ways forward.

Firstly, while those investigating the neurophysiology of breathlessness are now envisaging a multi-dimensional explanatory model based on that of pain, which takes the emotions into account, there remains a temporal linearity to this model that may obstruct creative thinking. Lancing and colleagues have represented their view of the perception of breathlessness in the model in Figure 16.1.

There is general agreement about this kind of conceptualisation, as Peiffer comments:

There is indeed increasing evidence that dyspnea encompasses affective/cognitive dimensions, including an immediate emotional reaction and a secondary, more sophisticated reaction ... which have a crucial influence on the subjective experience of this symptom. ${ }^{30}$

What is striking about this model from a critical medical humanities perspective is that affect is presented as deriving from the sensation of breathlessness, whereas the framework discussed above suggests that the experience of breathlessness is profoundly coloured by prior experience, beliefs and cultural influences. Thus affect is not just a response to being breathless, but also determines what that experience is like for the breathless person. Of course, models are not intended to replicate reality, as Annamaria Carusi says in her chapter in this volume, but nevertheless, this model strongly suggests that the neurophysiologists are not including the impact of prior emotional experience or belief amongst the afferents influencing the perception of breathlessness. 


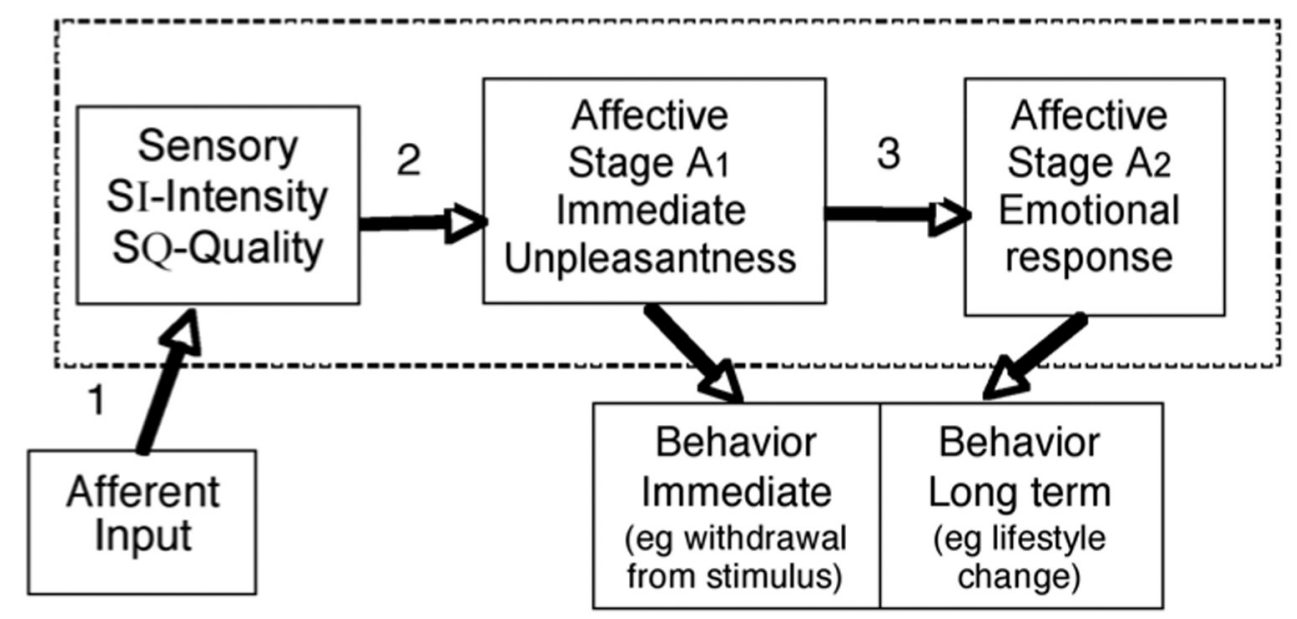

Fig. 2.

Definitions of the perceptual model (enclosed within dashed line)

Components of the Sensory Dimension: $\mathrm{SI}=$ Sensory Intensity, $\mathrm{SQ}=$ Sensory Quality; $\mathrm{SL}=$ Sensory location; ST = Time course of sensation

Components of the Affective Dimension: $A_{1}=$ Immediate Unpleasantness: first stage of the affective dimension; $\mathrm{A}_{2}=$ Emotion/Evaluation: second stage of affective dimension

Figure 16.1 Reprinted from The Multiple Dimensions of Dyspnea: Review and Hypothesis, Robert W. Lancing, Richard H. Gracely, Robert B. Bansett, 'Respiratory Physiology and Neurobiology', Fig. 2, p. 17. Copyright 2009, with permission from Elsevier.

\section{www.sciencedirect.com/science/journal/15699048}

What our work in the 'Life of Breath' project is directed towards is exploring the influence of space and place, the origins of beliefs and the nature of cultural influences on breath. The outcomes of this work, in dialogue with clinical collaborators, have the potential to enable us to influence the future shape of explanatory models by suggesting that initial sensory intensity and quality are affected by a range of prerequisites, not just physiological afferents. Understanding this and constructing a more accurate and complex model may in turn enable more effective hypotheses about management to be explored.

Secondly, the focus on pain as a road map might also lead to concentration on negative emotional correlates rather than the positive ones that may be associated with healthy breathlessness (of the kind people experience normally during exercise). In fact, this research has been carried out almost exclusively on healthy subjects because of the difficulty of subjecting breathless patients to prolonged scanning in a horizontal position; this leaves unanswered the question of what distinguishes non-pathological and pathological breathlessness in phenomenological and neurophysiological terms. This distinction has potential clinical importance because when patients and (healthy) health professionals talk about 'breathlessness', they may be referring to two distinctive and radically differing experiences of breathlessness. ${ }^{31}$

Our critical medical humanities approach proposes taking a step back to determine whether there are differences in the experience of these two groups. We will carry 
out comparative phenomenological work with what we call 'aware breathers', who develop an awareness of breathing because of certain practices, such as sport, singing or playing a wind instrument, and with people who have pathological breathlessness. We intend to investigate the differences between these two groups and discuss these with clinical collaborators. We hope to establish whether such differences might account for how pleasant or unpleasant breathlessness might appear differently in imaging studies. Such findings may enable more sophisticated predictive modelling of how breathlessness is mapped within the brain, as little work has been done to date to map normal breathing to allow for comparison.

Having approached breath and breathing from the perspective of the clinic, we now turn to the ways in which the humanities and social sciences have treated these phenomena. The next section focuses on the invisibility of breath. We conclude by considering how a novel method for making breath visible might provide a way of bringing humanities and clinical concerns fruitfully into dialogue.

\section{Stepping into the Invisible}

In both the humanities and social sciences, the theme of 'invisibility' looms large with respect to breathing. This theme is mirrored by Jennifer Richards and Richard Wistreich's discussion of the ephemeral nature of the voice in this volume. There is no developed phenomenological literature on breathing or breathlessness written from a philosophical perspective, although Havi Carel's work has begun to fill this lacuna; ${ }^{32}$ nor is there a comprehensive historico-cultural study of breathing. The closest cultural-historical analysis is Steven Connor's monograph, The Matter of Air, a study of human perceptions of air and how these have interacted with technology and culture. Connor provides insight into why breath has not been a significant object of study - precisely because (as he suggests of air):

How was one to make of the air such an object? How is the air to be picked out of its surroundings, when air was ambience itself? How was the air to be brought before one, when it was of necessity and at all times all about? ${ }^{33}$

In the clinical context, breathlessness' invisibility takes the form of millions of undiagnosed sufferers, hidden symptoms, stigmatised lives, and proxies being used for real (and therefore invisible) patients in clinical research. Within cultural theory, invisibility has a different interpretation. Echoing Connor's writing on air, Davina Quinlivan's monograph, The Place of Breath in Cinema, opens by saying: 'How can we start to think about something we cannot see? ${ }^{34}$ Both authors consider how the invisible can be made visible. For Connor, this includes interactions with machinery and the manufacturing of gasses; and for Quinlivan, a film studies scholar, it is through sound. She examines the laboured breath sounds of Darth Vader in Star Wars, and the 'terrible, troubled breathing' of John Merrick in The Elephant Man. ${ }^{35}$

Quinlivan is interested in a broader investigation of breath in relation to theories of the body in relation to the arts. Her monograph explores 'how breathing represents a 
subtle dimension of our bodies that can be seen to be both inside and outside of ourselves'. ${ }^{36}$ We add that breathing not only is both 'inside' and 'outside' ourselves, but also acts as a conduit and a connection with the world around us. This connection is physical; it is literally air we inhale from the outside that becomes incorporated into our bodies in the form of oxygen molecules in the blood stream. But it is also a spiritual connection, relating us to the symbolic or non-material context.

For example, David Abram, in The Spell of the Sensuous, discusses how the Navajo tribe consider breathing an act of keeping in contact with spirits, which are in the air and can be connected with via respiration. ${ }^{37}$ Abram writes that, according to the Navajo, 'this invisible medium, in which we are bodily immersed, is what provides us with the capacity for conscious thought. ${ }^{38}$ Such ideas resonate with notions from ancient Greek texts that are deeply embedded in our culture. Aristotle, in his treatise 'On Respiration', speaks of an extended mind or soul that is part of the surrounding air and enters the body with the intake of breath. ${ }^{39}$

This connection between wind/air and spirit is noted etymologically in many languages; from the Sanskrit Atman to the Hebrew Ruach and the Greek pneuma, we find that the terms for air, breath and spirit overlap. ${ }^{40}$ Closer to home, we might consider the intuitive act of inhaling deeply when getting to the seaside or countryside. Breathing in the fresh air might act to remove some of the city pollutants (exhaust fumes, industrial pollution) but also, on a psychological or spiritual level, it may mean taking in the calm of the countryside, to replace the hectic city stress.

This reflection and the desire to investigate the way in which a study of breath can further illuminate theories of the body lead to phenomenological exploration in the context of a medical anthropology study of 'aware breathers' described above. Simone Denis, in her ethnography of smokers, describes 'Megan' using her smoky breath to flirt. She quotes Megan as saying, 'If I am interested [in a man] I like to blow my smoke up around the side of his face, like a caress. ${ }^{41}$ The cigarette smoke - the visible expulsion of breath - is used by her in a sensuous way to 'touch' the other person.

Brian Lande explores another group of aware breathers in his ethnography of soldiers. His study demonstrates the importance of 'breathing like a soldier' for two crucial activities: running and shooting. Keeping up with the leaders running long distances is a prerequisite for military activity. In order to maintain authority, the troop leader must not be found 'puffing at the rear'. Firing a rifle requires understanding of how breathing might nudge the sights off target. Lande concludes: 'breathing is far from being a taken-for-granted physical activity. It is the social sinew that holds together social institutions by anchoring norms and beliefs in viscera. ${ }^{42}$ As noted in the previous section, there has been little ethnographic work with 'aware breathers', but the few existing studies indicate the central role of breath in identity formation and sense of self within the world. Importantly, these identities are not necessarily continuous or even overlapping with those supplied, learned or assumed within the clinical context.

There are also 'aware breathers' who are aware of their breath because it is pathological. They experience breath as a lack, insufficiency or absence. In such cases the 
experiences of breathlessness incorporate feelings of fear or impending death, for example, as is reported by patients who are severely short of breath. However, these experiences are discontinuous with the clinical account, which focuses on measuring objective lung function and on assessing functionality with respect to daily living, rather than the subjective experience of breathlessness. ${ }^{43}$ The protagonist in Michael Symmons Roberts's novel, Breath, reflects upon his breathing while he awaits a lung transplant, after his lungs were damaged in a gas attack:

Somehow, even now on the brink of having his weakest lung cut out and replaced with a new one, he can't locate the problem in his own chest. Sure his chest is heaving as his lungs try to drag in the air, but it still feels like a problem with the air, not with his body. On that April morning so many years ago, the air itself was altered, and his sensitive lungs failed to adapt. ${ }^{44}$

The focus of this aware breather is on the air failing to provide what his body craves. It is the medium within which he exists that is now in some way alien to his body. He is like a fish out of water, unable to access what he needs from the surrounding element.

The importance of the air as a medium is signalled in the first episode of the BBC's Doctor Who starring Peter Capaldi as the Doctor, entitled 'Deep Breath' ${ }^{45}$ In a pivotal and uncanny scene, the Doctor and Clara, his assistant, meet in a restaurant surrounded by other diners. The Doctor becomes aware of an eerie stillness, and plucks one of Clara's long hairs to test for movement in the air. He twists the hair round his finger and drops it. The hair falls to the ground without deviation and the Doctor concludes that none of the other diners is breathing and thus there is no air movement. The uncanniness of this scene contrasts deeply with the Navajo tribe's conception of air as a living, constantly moving, connecting medium, which enters one body and then reunites with the atmosphere, only to enter another body. As McNeley writes:

According to the Navajo conception [... .] Winds exist all around and within the individual, entering and departing through respiratory organs and whorls on the body's surface. That which is within and that which surrounds one is all the same and it is holy. ${ }^{46}$

These examples from contemporary and historical cultural contexts draw attention away from the body as the site of the problem to the surrounding medium, the air, and suggest that more work outside a clinical context might be fruitful, to explore the perceptions of the air by aware breathers as well as by those who suffer breathlessness. The champion free-diver, Guillaume Néry, regularly experiences complete absence of the ability to breathe within the medium of water when he undertakes deep dives that expose him to carbon dioxide narcosis. This experience is powerfully portrayed in a film by Julie Gautier, in which Néry is seen descending into the darkness of the sea, propelling himself by a large seal-like flipper. ${ }^{47}$ As Néry descends, the viewer is overcome with a feeling of panic and fear that is felt physically as a breath-holding experience. The film portrays Néry's hallucinatory experiences while in this breathless 
medium and this takes the viewers into a different mode of being, as we forget the need to hold our breath, until he starts to swim upwards again.

These examples illustrate how culture can both portray and evoke experience, as it holds a wealth of sources that not only inform but also reflect how people experience breathlessness, both pathological and non-pathological. Understanding these sources, analysing them, and unpicking their connection to and impact on the clinic is the task of our 'Life of Breath' project.

How might some of these ideas help us in this task? At the time of writing, our work is only just starting but initial interdisciplinary discussions are beginning to bear fruit, in particular regarding the need for non-narrative accounts of breathing and breathlessness, as these seem to be almost entirely missing from the cultural corpus. We now turn to a second case study illustrating critical medical humanities in action.

\section{Bridging the Epistemic Gap 2: Visualising Breath}

At the core of the project is a research group: 'Breathing Space'. This group includes academics from humanities and social science disciplines, as well as clinicians, health service researchers, artists and designers, and is run by a creative facilitator whose role is to ensure democracy and sharing of knowledge, skills and methods. The 'space' enabled by the meetings is intended to be creative: to enliven ideas in individuals and subgroups that may be taken forward to progress the aims of the project. A recent meeting (January 2015) gave rise to a potentially exciting idea.

This meeting featured a talk by artist Jayne Wilton, whose work has focused on attempting to make breath visible..$^{48}$ This talk, and our group reflection on how Jayne's work might be not only aesthetically appreciated but also clinically useful, stimulated a design colleague, David Swann, to investigate whether it might be possible to use a breath visualisation technique in the clinic. The technique now investigated is the 'shadowgraph'. This is a simple technique that does not require the use of irritants or toxic tracers or intense lighting to have its effects. Breath visualisation is achieved with the use of a spherical, concave, high-precision mirror with a relatively low-voltage white-light source and a high-speed digital camera. The person whose breath is visualised stands in front of the mirror and images are obtained when reflected light from the mirror is refracted to different degrees as it passes from the warmth of the mouth to the cooler surrounding air. ${ }^{49}$ The image that results looks like the surface of the moon, billowing out in particular patterns. Measurements can be made of the breath cloud relating to distance travelled, exhaled velocity, expansion rate and direction of flow. As Tang et al. discuss, it has been used largely to explore how far exhaled breath extends as a guide to aiding aerosol infection control. ${ }^{50}$

Such a technique has several potential applications. Primarily, it answers what seems, from the cultural references we have explored above, to be a desire to make visible what has been invisible, and to understand better the shape and form of breath in the air. People who experience breathing difficulties echo Michael Symmons Roberts's character in Breath, remarking on the ways in which the ambient air, its texture and weight, affect their breathing. ${ }^{51}$ With this technique we might explore how the ability 
to see breath might help those with breathing difficulties to understand and articulate this relationship and explain it more clearly to their clinicians. It may also provide a new and non-invasive method for measuring breath, especially if particular patterns of the breath cloud relating to distinct conditions could be described.

This thinking would not have been possible without the interdisciplinary aegis of our project. Testing its applicability and usefulness will require our clinical and artistic collaborators, working with a designer and medical humanities lead. The idea has the potential to influence patients, provide stimulus for discussion amongst our 'aware breather' cohorts, and engage the public who may learn from seeing their breath in action.

\section{Conclusion}

This chapter opened by signposting the work we intend to carry out in the next five years. It is an invitation for the reader to reflect on and engage with the ideas presented here, which we will continue to develop. The principal idea of a critically engaged 'helping' medical humanities stands at the core of this chapter and our work. We see breath as a fruitful field of exploration, a domain where important areas of clinical need have been identified and which our fields can help answer. But this is only one part of the work to be done. We also hope to contribute in a variety of ways to understanding the phenomenology of the body and of breathing, and their exploration in art and culture.

The question of what critical medical humanities may wish to achieve is partly a scholarly one, as this collection demonstrates, but we also maintain that it has an important ethical dimension. If the fields of medical humanities, humanities and social sciences have knowledge that may contribute to the care of breathless people, there seems to be a moral imperative to engage with clinicians and biomedical researchers on this topic. It is our goal to pursue ways of improving the understanding of breathlessness not only in our fields but also in the clinic.

\section{Further Reading}

David Abram, The Spell of the Sensuous (London: Vintage Books, 1996).

British Lung Foundation, Invisible Lives: Chronic Obstructive Pulmonary Disease (COPD) Finding the Missing Millions (London: British Lung Foundation, 2007).

Havi Carel, Illness (London: Routledge, 2013).

Havi Carel, Phenomenology of Illness (Oxford: Oxford University Press, 2016).

Steven Connor, The Matter of Air: Science and the Art of the Ethereal (London: Reaktion Books, 2010).

Marjolein Gysels and Irene J. Higginson, 'Access to Services for Patients with Chronic Obstructive Pulmonary Disease: The Invisibility of Breathlessness', Journal of Pain and Symptom Management 36.5 (2008), pp. 451-60.

Mari Herigstad, Anja Hayen, Katja Wiech and Kyle T. S. Pattinson, 'Dyspnoea and the Brain', Respiratory Medicine 105.6 (2011), pp. 809-17.

Megan Wainright and Jane Macnaughton, 'Is a Qualitative Perspective Missing from COPD Guidelines?’ Lancet Respiratory Medicine 1.6 (2013), pp. 441-2. 


\section{Notes}

1. The 'Hearing the Voice' project is based at Durham University and is an interdisciplinary exploration of the experience of hearing a voice in the absence of external stimuli. See <https://www.dur.ac.uk/hearingthevoice/> (accessed 1 September 2014).

2. The 'Life of Breath' project is funded by the Wellcome Trust from 2014 to 2019, based at Durham and Bristol universities, and led by the authors.

3. Angela Woods, 'The Limits of Narrative: Provocations for Medical Humanities', Medical Humanities 37.2 (2011), pp. 73-8.

4. Ibid.

5. Anne Whitehead, 'The Medical Humanities: a Literary Perspective', in Victoria Bates, Alan Bleakley and Samuel Goodman (eds), Medicine, Health and the Arts: Approaches to the Medical Humanities (London: Routledge, 2014), pp. 107-27.

6. Daniel Fox, 'Who We Are: The Political Origins of the Medical Humanities', Theoretical Medicine 6 (1985) pp. 327-41 (p. 329).

7. Jane Macnaughton, 'Medical Humanities Challenge To Medicine', Journal of Evaluation in Clinical Practice 17 (2011), pp. 927-32.

8. Dermot Moran, 'Edmund Husserl's Phenomenology of Habituality and Habitus', Journal of the British Society for Phenomenology 42.1 (2011), pp. 53-77 (p. 56).

9. Havi Carel and Ian James Kidd, 'Epistemic injustice in healthcare: A philosophical analysis', Medicine, Healthcare and Philosophy 17:4 (2014), pp. 529-40. DOI 10.1007/s11019014-9560-2.

10. William James, The Varieties of Religious Experience (London: Longmans Green and Co., 1929), p. 16.

11. Megan Wainright and Jane Macnaughton, 'Is a Qualitative Perspective Missing from COPD Guidelines?’, Lancet Respiratory Medicine 1.6 (2013), pp. 441-2.

12. Charlotte A. Roberts, 'A Bioarcheological Study of Maxillary Sinusitis', American Journal of Physical Anthropology 133 (2007), pp. 792-807.

13. Peter J. Barnes and Sabine Kleinert, 'COPD - A Neglected Disease', Lancet 364 (2014), pp. 564-5.

14. British Lung Foundation, Invisible Lives: Chronic Obstructive Pulmonary Disease (COPD) Finding the Missing Millions (London: British Lung Foundation, 2007), p. 3.

15. Marjolein Gysels and Irene J. Higginson, 'Access to Services for Patients with Chronic Obstructive Pulmonary Disease: The Invisibility of Breathlessness', Journal of Pain and Symptom Management 36.5 (2008), pp. 451-60.

16. Ibid., pp. 455-6.

17. Peter J. Barnes and Sabine Kleimert, 'COPD - a neglected disease', pp. 564-5.

18. Mark B. Parshall, Richard M. Schwartztein, Lewis Adams, Robert B. Banzett, Harold L. Manning, Jean Bourbeau, Peter M. Calverley, Audrey G. Gift, Andrew Harver, Suzanne C. Lareau, Donald A. Mahler, Paula M. Meek and Denis E. O’Donnell; on behalf of the ATS Committee on Dyspnea, 'An Official American Thoracic Society Statement: Update on the Mechanisms, Assessment, and Management of Dyspnea', American Journal of Respiratory and Critical Care Medicine 185.4 (2012), p. 445.

19. Miriam J. Johnson, David C. Currow and Sara Booth, 'Prevalence and Assessment of Breathlessness in the Clinical Setting', Expert Review of Respiratory Medicine 8.2 (2014), pp. 151-61.

20. Parshall et al., 'An Official American Thoracic Society Statement', pp. 435-52. 
21. Ibid., p. 444.

22. Mari Herigstad, Anja Hayen, Katja Wiech and Kyle T. S. Pattinson, 'Dyspnoea and the Brain', Respiratory Medicine 105.6 (2011), pp. 809-17 (p. 810).

23. Ibid., p. 810.

24. Ibid., p. 813.

25. Claudine Peiffer, 'Dyspnea Relief: More Than Just the Perception of a Decrease in Dyspnea', Respiratory Physiology and Neurobiology 167 (2009), pp. 61-71.

26. Herigstad et al., 'Dyspnoea and the Brain', p. 814.

27. Parshall et al., 'An Official American Thoracic Society Statement', p. 445.

28. Herigstad et al., 'Dyspnoea and the Brain', p. 811.

29. Robert W. Lansing, Richard H. Gracely and Robert B. Banzett, 'The Multiple Dimensions of Dyspnea: Review and Hypotheses', Respiratory Physiology and Neurobiology 167 (2009), pp. 53-60.

30. Ibid., p. 62.

31. Havi Carel, Illness (London: Routledge, 2013).

32. Ibid. See also Phenomenology of Illness (Oxford: Oxford University Press, 2016).

33. Steven Connor, The Matter of Air: Science and the Art of the Ethereal (London: Reaktion Books, 2010), p. 17.

34. Davina Quinlivan, The Place of Breath in Cinema (Edinburgh: Edinburgh University Press, 2012), p. 1.

35. Ibid., pp. 4-5.

36. Ibid., p. 2.

37. David Abram, The Spell of the Sensuous (London: Vintage Books, 1996).

38. Ibid., pp. 233-4.

39. Aristotle, On the Soul, Parva Naturalia, On Breath, trans. W. S. Hett (London: Heinemann, 1957), p. 439.

40. Abram, The Spell of the Sensuous, pp. 237-8.

41. Simone Denis, 'Smoking Causes Creative Responses: On State Antismoking Policy and Resilient Habits', Critical Public Health 21.1 (2011), pp. 25-35.

42. Brian Lande, 'Breathing like a Soldier: Culture Incarnate', Sociological Review 55 (s1) (2007), pp. 95-108 (p. 97).

43. Herigstad et al., 'Dyspnoea and the Brain', p. 810.

44. Michael Symmons Roberts, Breath (London: Vintage Books, 2009), p. 103.

45. Dr Who: Deep Breath, television, produced and written by Steven Moffat. UK: BBC Television, first broadcast 23 August 2014.

46. McNeley, cited in Abram, The Spell of the Sensuous, p. 233.

47. Narcose, film, directed by Julie Gautier <http://io9.com/experience-the-hallucinations-ofa-freedivers-carbon-di-1615995489> (accessed 7 September 2014).

48. Jayne Wilton, Breathe and Drawing Breath <http://www.jaynewilton.com/gallery.html> (accessed 16 March 2015).

49. Julian W. Tang, Andrew Nicolle, Jocan Panetelic, Gerald C. Koh, Liang De Wang, Muhammad Amin, Christian A. Klettner, David K. W. Cheong, Chandra Sekhar, Kwok Wai Tham, 'Airflow Dynamics of Coughing in Healthy Human Volunteers by Shadowgraph Imaging: an Aid to Aerosol Infection Control', PLoS ONE 7.4 (2012), e34818.

50. Ibid.

51. Content of discussion with a member of the British Lung Foundation's 'Breathe Easy' Group (local group in North-east of England, March 2015). 